\title{
What makes a human brain?
}

The quest to identify what makes humans so different from our closest relatives, the great apes, focuses largely on our brains. What has allowed us to develop such enlarged brains, and such intellectual ability, when compared with our primate cousins? A new study looks for answers in the transcriptomes - the patterns of mRNA expression - in the brains of humans, chimpanzees and macaques.

It has become increasingly clear over recent years that the genome is not everything when it comes to defining an animal. It is also essential to understand where, when and to what extent genes are expressed during development and in the mature animal. So to look at what drives the differences between primate brains, Konopka et al. used advanced sequencing and microarray techniques to measure the expression of mRNA transcripts in three areas of the brain - the caudate nucleus, the hippocampus and the frontal pole. The resulting transcriptome provides insight into the spatial patterns of gene expression in these brain areas and allows comparisons between the three species.

Comparisons of the lists of genes that are expressed in each area identified genes that were differentially expressed in the human brain when compared with the macaque or chimpanzee brain, and there were more differentially expressed genes in the human frontal pole than in the caudate nucleus or hippocampus. This is perhaps not surprising, as the human frontal cortex is the most disproportionately enlarged part of the brain and is thought to be responsible for many of the functions that distinguish our cognitive skills from those of other primates. The genes identified include several that have been implicated in neuronal maturation, differentiation and function.

By contrast, gene expression patterns in the caudate nucleus were much more similar among the human, chimpanzee and macaque brains. This might reflect more conserved functions for this part of the brain.

Konopka et al. carried out further analyses on the data using a technique called weighted gene co-expression network analysis. This allowed the authors to define 'modules' consisting of networks of genes in which expression was correlated (positively or negatively) in a particular area. There were more modules related to the frontal pole than to the other areas in the human brain, but not in the chimpanzee or macaque brains, and the authors interpret this as showing that the human frontal pole has greater transcriptional complexity than the chimpanzee or macaque frontal poles.

In their paper, Konopka et al. focus on some specific modules that centre around genes of particular interest. One example contains several genes that have been implicated in neuropsychiatric disorders and seems to have at its core the circadian rhythm gene CLOCK. Another contains the forkhead transcription factor FOXP2, which is linked to speech and language functions in humans, and the genes that comprise this network are more interconnected in humans than in chimpanzee and macaque brains.

In an accompanying commentary, Ponting and Oliver discuss these results and summarize some of the difficulties in interpreting them. For example, the levels of mRNA (as measured in this study) do not always correlate with the levels of protein expression. Despite these limitations, Konopka et al. have expanded our understanding of gene expression patterns in the brain and shed new light on human brain evolution.

Rachel Jones

ORIGINAL RESEARCH PAPER Konopka, G. et al. Human-specific transcriptional networks in the brain. Neuron 75, 601-617 (2012) FURTHER READING Ponting, C. P. \& Oliver, P. L. Brain, know thy transcriptome, know thyself. Neuron 75, 543-545 (2012)

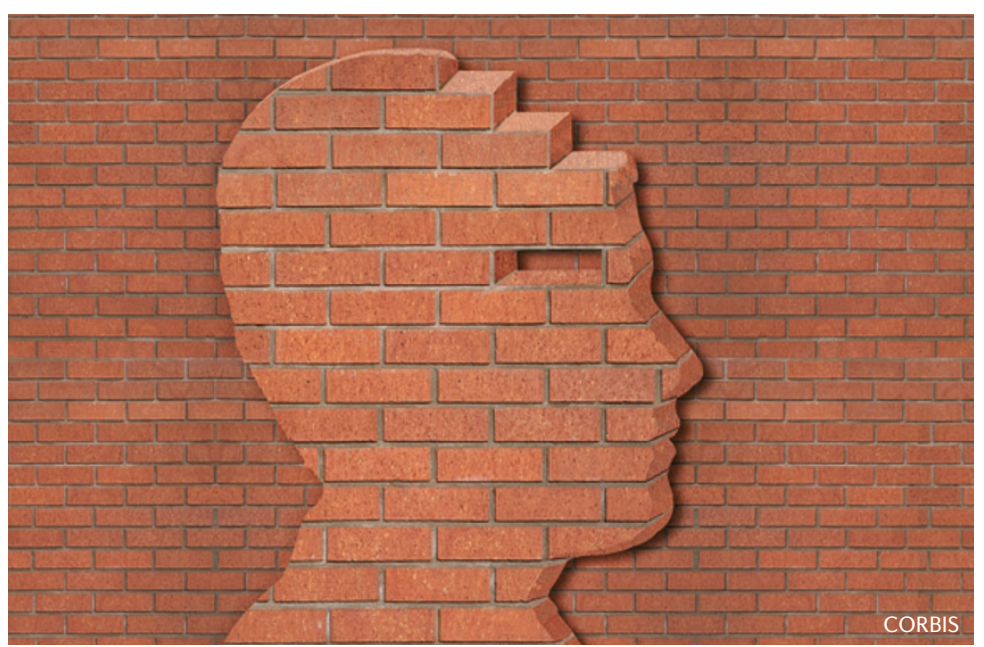

